\title{
ON THE OPTIMUM ABSORBER PARAMETERS: REVISING THE CLASSICAL RESULTS
}

\author{
VOLODYMYR PUZYROV \\ Donetsk National University, Department of Higher Mathematics and Methodology of Teaching, Vinnytsya, Ukraine \\ e-mail: doe.seldon@gmail.com \\ JAN AWREJCEWICZ \\ Lodz University of Technology, Department of Automation, Biomechanics and Mechatronics, Eódź, Poland \\ e-mail: awrejcew@p.lodz.pl
}

\begin{abstract}
The dynamic vibration absorber is a kind of mechanical device with inertia, stiffness, and damping. Once connected to a given structure or machine, it is capable of absorbing vibratory energy. As a result, the primary system can be protected from excessively high vibration levels. In this paper, we deal with classical Den Hartog's model to clarify the known results and improve the mathematical component of this approach. We suggest the optimal choice of absorber parameters, which is slightly different and more general analytical approach. The comparison of two methods of optimization is carried out, and the corresponding error of calculus is estimated.
\end{abstract}

Keywords: dynamic vibration absorber, Den Hartog's model, frequency-amplitude curve, optimization

\section{Introduction}

The problem of elimination or reduction of undesired vibration in various technical systems has long history and great achievements. The concept of vibration control is widely accepted nowadays and has been applied in many different areas, such as civil, mechanical, and aeronautical engineering. Passive vibration control is the most widespread, and effective methods are available. The typical simplest and most reliable device is the dynamic vibration absorber (DVA) or tuned-mass damper (TMD). A simple DVA consists of a mass and a spring. When the primary system is excited by a harmonic force, its vibration can be suppressed by attaching a DVA. The main purpose of adding the secondary oscillator is to move the resonant frequency of the mechanical system away from the operating frequency of the vibratory force. So, the system becomes a 2-DOF (degree of freedom) mechanical system, but with coincidence of the exciting frequency with one of the two natural frequencies it will be again at resonance. To eliminate this effect, a damper is added to the DVA (Fig. 1).

The idea of vibration control was originally proposed by Frahm (1911), and many various DVA configurations were designed during the past century (Ormondroyd and Den Hartog, 1928; Brock, 1946; Mead, 2000; Hunt, 1979; Korenev and Reznikov, 1993; Viet et al., 2011; Marano et al., 2007). Good surveys on the subject were presented in (Johnson, 1995; Sun et al., 1995). With reference to the DVA optimal design, the first criterion was offered by Ormondroyd and Den Hartog (1928). This criterion concerned the minimization of the system response with respect to the stationary harmonic excitation with the most "dangerous" frequency value which results in the largest increase of the amplitude. 


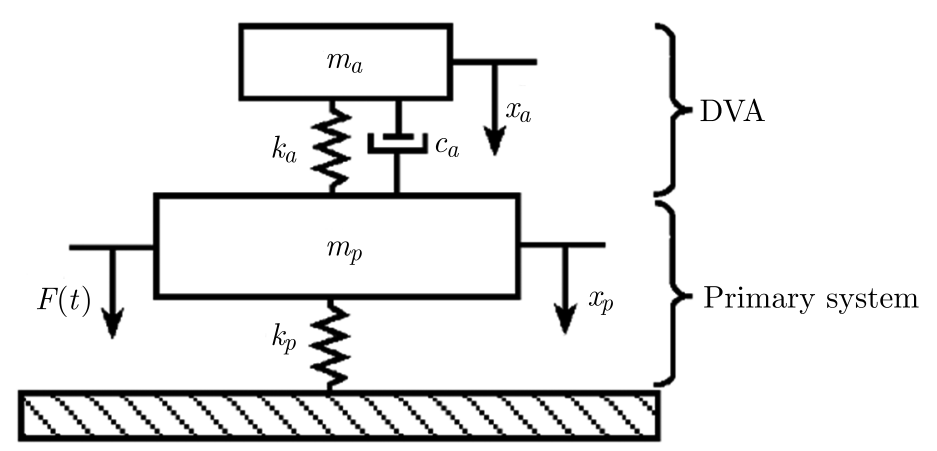

Fig. 1. Damped DVA connected to a primary system

\section{Formulation of the problem}

The equations of motion of the mechanical system under consideration are

$$
\begin{aligned}
& m_{p} \ddot{x}_{p}+c_{a}\left(\dot{x}_{p}-\dot{x}_{a}\right)+k_{p} x_{p}+k_{a}\left(x_{p}-x_{a}\right)=F_{0} \mathrm{e}^{\mathrm{i} \omega t} \\
& m_{a} \ddot{x}_{a}+c_{a}\left(\dot{x}_{a}-\dot{x}_{p}\right)+k_{a}\left(x_{a}-x_{p}\right)=0
\end{aligned}
$$

In this formulation, we use the notions according to (Johnson, 1995), $F(t)=F_{0} \mathrm{e}^{\mathrm{i} \omega t}$ is a harmonic excitation force acting on the primary system.

The amplitudes of steady state harmonic responses are

$$
\begin{aligned}
& X_{p}=F_{0} \frac{k_{a}-m_{a} \omega^{2}+\mathrm{i} \omega c_{a}}{\left(k_{p}-m_{p} \omega^{2}\right)\left(k_{a}-m_{a} \omega^{2}\right)-m_{a} k_{a} \omega^{2}+\mathrm{i} \omega c_{a}\left(k_{p}-m_{p} \omega^{2}-m_{a} \omega^{2}\right)} \\
& X_{a}=-F_{0} \frac{k_{a}+\mathrm{i} \omega c_{a}}{\left(k_{p}-m_{p} \omega^{2}\right)\left(k_{a}-m_{a} \omega^{2}\right)-m_{a} k_{a} \omega^{2}+\mathrm{i} \omega c_{a}\left(k_{p}-m_{p} \omega^{2}-m_{a} \omega^{2}\right)}
\end{aligned}
$$

In terms of dimensionless parameters, we can rewrite

$$
\frac{\left|X_{p}\right|}{\left(X_{p}\right)_{s t}}=\sqrt{\frac{(2 \zeta g)^{2}+\left(g^{2}-\eta^{2}\right)^{2}}{(2 \zeta g)^{2}\left(g^{2}-1+\mu g^{2}\right)+\left[\mu \eta^{2} g^{2}+\left(g^{2}-1\right)\left(g^{2}-\eta^{2}\right)\right]}}
$$

where $\mu=m_{a} / m_{p}$ is the mass ratio; $\omega_{a}=\sqrt{k_{a} / m_{a}}$ - undamped natural frequency of the DVA considered separately; $\omega_{p}=\sqrt{k_{p} / m_{p}}$ - undamped natural frequency of the primary system considered separately; $\eta=\omega_{a} / \omega_{p}$ - tuning factor; $g=\omega / \omega_{p}$ - forcing frequency ratio; $\zeta=c_{a} /\left(2 m_{a} \omega_{p}\right)$ - damping ratio; $\left(X_{p}\right)_{s t}=F_{0} / k_{p}$ - static displacement of the primary mass.

Many methods of optimization have been developed to opportunely design this vibration control technique. In the classical textbook on mechanical vibrations, Den Hartog (1940) pointed out a remarkable feature: for any fixed values of $\eta$ and $\mu$, curves (2.3) intersect in two points $P$ and $Q$ (named "invariant points"), as shown in Fig. 2, independently of the value of $\zeta$. These points are situated close enough to the peaks of the frequency-amplitude curve. Den Hartog suggested to choose the parameter $\eta$ to equalize ordinates of $P$ and $Q$. Secondly, $\zeta$ was taken to satisfy the condition of "almost horizontal" tangents in the invariant points. Thus the values

$$
\eta_{\text {opt }}=\frac{1}{1+\mu} \quad \zeta_{\text {opt }}=\sqrt{\frac{3 \mu}{8(1+\mu)^{3}}}
$$

were obtained. 


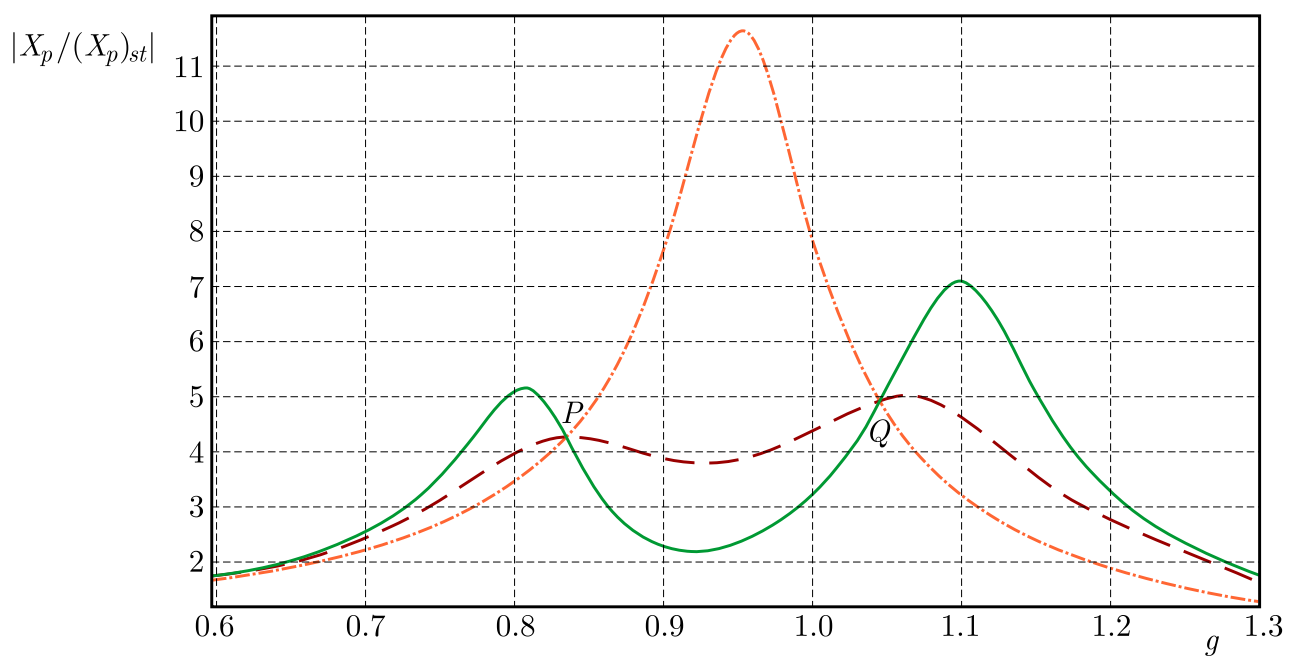

Fig. 2. Invariant points for $\eta=0.9, \mu=0.1$. Solid line $\zeta=0.027$, long dash line $\zeta=0.16$, dot-dash line $\zeta=0.5$

\section{Mathematical analysis of the problem}

The approach of Den Hartog has undoubted advantages: simplicity, which is essential for applied researchers, and rather high accuracy, as was shown in numerical simulations. At the same time, it is possible to note two drawbacks of this approach. The first one is the absence of analytical assessment of the difference between the maximum response value proposed and the true value. The second is that the scheme of determining the optimal values is based on the existence of invariant points, which is an exception rather than the rule. For example, this approach does not fit in the case when the main body is damped itself (Warbrton and Ayorinde, 1980), or for sky-hooked (Griffin et al., 2002; Liu and Liu, 2005) DVA. So, in such cases, one have to rely upon numerical methods for optimization. It should be added that from the theoretical viewpoint, the question on the existence of the exact solution of the problem in an algebraic form is not closed.

Considering the above-mentioned, our aims are: 1) to provide a more general approach which does not rely on exclusive properties of (2.3) (invariant points existence); 2) to evaluate the error of formulas (2.4).

At the beginning, let us consider a function

$$
f(\mu, \delta, h, \gamma)=\frac{h \gamma+(\gamma-\delta)^{2}}{h \gamma(\gamma+\mu \gamma-1)^{2}+[\mu \delta \gamma-(\gamma-1)(\gamma-\delta)]^{2}}
$$

which we believe is more suitable for the analysis. Here

$$
\delta=\eta^{2} \quad h=4 \zeta^{2} \quad \gamma=g^{2} \quad f=\left(\frac{\left|X_{p}\right|}{\left(X_{p}\right)_{s t}}\right)^{2}
$$

Obviously, the optimization of function (2.3) is the same as function (3.1). The optimal values from (2.4) in the new notions are

$$
\delta=\frac{1}{(1+\mu)^{2}} \quad h=\frac{3 \mu}{2(1+\mu)^{3}}
$$

The typical shapes of the surface $f(\gamma, \mu)$ with fixed values $\delta, h$ are presented in Fig. 3 . 

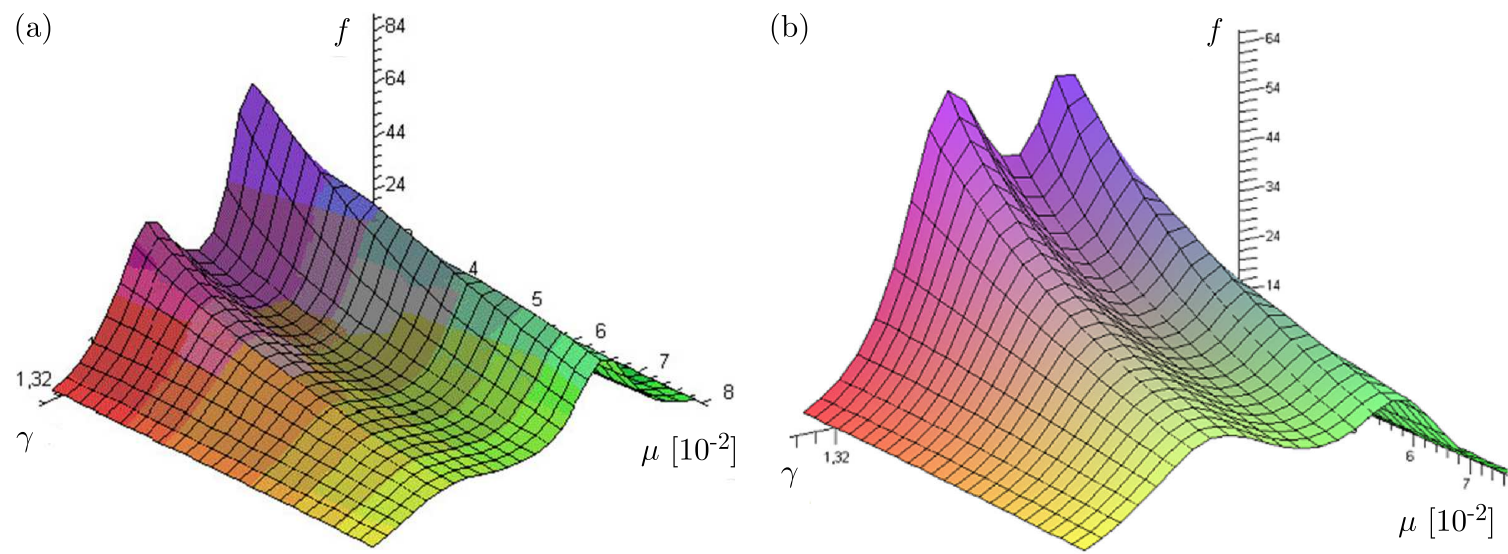

Fig. 3. Typical character of surface (3.1): (a) $\delta=1-2.5 \mu, h=0.5$, (b) according to Eqs. (3.3)

It is remarkable that for any given set of $(\delta, h, \mu)$, zeros of the derivative $d f / d \gamma$ lead to the following equation

$$
\begin{aligned}
\gamma^{5}- & \gamma^{4}\left[4 \delta-2 h+\mu(\delta-h)-\frac{1}{2} h \mu^{2}\right]+\gamma^{3}\left[6 \delta^{2}+4 \delta-4 h \delta+h^{2}-2 h+2 \mu\left(2 \delta^{2}-3 h \delta+h^{2}\right)\right. \\
& \left.-h \mu^{2}(2 \delta-h)\right]-\gamma^{2}\left[4 \delta^{3}-2 h \delta^{2}+6 \delta^{2}-4 h \delta+h^{2}+\mu\left(5 \delta^{3}-4 h \delta^{2}+\delta^{2}-3 h \delta+h^{2}\right)\right] \\
& +\gamma \delta^{2}\left[\delta^{2}+4 \delta-2 h+2 \mu\left(\delta^{2}+\delta-h\right)+\mu^{2} \delta^{2}\right]-\delta^{4}(1+\mu)=0
\end{aligned}
$$

This equation of the 5-th order cannot be solved in an explicit form and, therefore, there is no way to make a conclusion on the maximum value of $f$. Because of this, we require an indirect method to achieve our goal. ${ }^{1}$

Let $f_{0}=1 / \kappa$ be some fixed number. Then the equation $f=f_{0}$ is equivalent to the following polynomial equation

$$
\begin{aligned}
& u(\gamma)=\gamma^{4}+\left(h+h \mu^{2}+2 h \mu-2-2 \delta-2 \mu \delta\right) \gamma^{3}+\left(\mu^{2} \delta^{2}+4 \delta-2 h+2 \mu \delta-2 h \mu+1\right. \\
& \left.+\delta^{2}-\kappa+2 \mu \delta^{2}\right) \gamma^{2}+\left(h-2 \delta^{2}-2 \mu \delta^{2}+2 \delta \kappa-2 \delta-h \kappa\right) \gamma+\delta^{2}-\delta^{2} \kappa=0
\end{aligned}
$$

In the case when curve (3.1) has two peaks, for some values of $\kappa$ the line

$$
f=\frac{1}{\kappa}
$$

intersects this curve four times, and equation (3.5) has four real positive roots. Otherwise, when line (3.6) is above curve (3.1), equation (3.5) has no positive roots. On the assumption that both peaks have the same height, we conclude that this is a borderline between two cases. In other words, the discriminant of the polynomial $u(\gamma)$ is equal to zero, and (3.5) has two pairs of multiple roots. Thereby, there exist such expressions $M(\mu, \delta, h), N(\mu, \delta, h)$ that

$$
u(\gamma)=\left(\gamma^{2}+M \gamma+N\right)^{2} \quad M^{2}-4 N>0
$$

Then, we conclude from (3.7) that

$$
\begin{aligned}
& \left(-2 \delta+2 \mu h+\mu^{2} h-2 \mu \delta-2 M-2+h\right) \gamma^{3} \\
& \quad+\left(1+2 \mu \delta^{2}+2 \mu \delta-2 h-\kappa+4 \delta+\delta^{2}-2 \delta N-2 \mu h+\mu^{2} \delta^{2}-M^{2}\right) \gamma^{2} \\
& \quad+\left(-2 \delta^{2}-h \kappa+2 \delta \kappa-2 \delta M N-2 \delta+h-2 \mu \delta^{2}\right) \gamma+\delta^{2}(1-\kappa)-N^{2}=0
\end{aligned}
$$

\footnotetext{
${ }^{1}$ An approach based on investigation of equation (3.4) with Taylor expansions representation was presented in (Pozdniakovich and Puzyrov, 2009). It allowed one to achieve some progress, comparatively with (3.3), but again, no explicit form and error estimation were gained.
} 
Now we have a system of four equations with five variables $\kappa, \delta, h, M, N$, and can consistently find

$$
\begin{aligned}
& \kappa=1-\left(\frac{N}{\delta}\right)^{2} \quad h=\frac{2[(1+\mu) \delta+M+1]}{(1+\mu)^{2}} \\
& M=-\frac{\delta^{2}(1+\mu)^{3}+\mu \delta N^{2}(1+\mu)-N^{2}}{N\left[\delta(1+\mu)^{2}-N\right]}
\end{aligned}
$$

The last equation is

$$
\begin{aligned}
& (\delta-N)\left[\delta^{9}(1+\mu)^{6}+\delta^{8} N(1+\mu)^{6}-4 \delta^{7} N(1+\mu)^{4}-4 \delta^{6} N^{2}(1+\mu)^{4}\right. \\
& \quad+2 \delta^{5} N^{2}(1+\mu)^{2}(3+\mu)+2 \delta^{4} N^{3}(1+\mu)^{2}(3+\mu)-4 \delta^{3} N^{3}(1+\mu) \\
& \left.\quad-2 \delta^{2} N^{4}\left(2+2 \mu+\mu^{2}\right)+\delta N^{4}+N^{5}\right]=0
\end{aligned}
$$

Condition (3.9) gives an equation of the 9 -th order on $\delta$ (and 5 -th order on $N$ ), but with the substitution

$$
\delta=\frac{\delta_{1}}{(1+\mu)^{2}} \quad N=\frac{N_{1} \delta_{1}^{2}}{(1+\mu)^{2}}
$$

it may be rewritten as

$$
\begin{aligned}
& N_{1}^{2} \delta_{1}^{2}\left[N_{1}^{4}(1+\mu)^{2}-2 N_{1}^{3}\left(2+2 \mu+\mu^{2}\right)+2 N_{1}^{2}(3+\mu)-4 N_{1}+1\right]+2 N_{1}^{4} \mu^{2} \delta_{1} \\
& -\left[N_{1}^{4}(1+\mu)^{2}-4 N_{1}^{3}(1+\mu)+2 N_{1}^{2}(3+\mu)-4 N_{1}+1\right]=0
\end{aligned}
$$

The last one being factorized as

$$
\begin{aligned}
& \left(N_{1} \delta_{1}-1\right)\left\{\delta_{1}\left[N_{1}^{4}(1+\mu)^{2}-2 N_{1}^{3}\left(2+2 \mu+\mu^{2}\right)+2 N_{1}^{2}(3+\mu)-4 N_{1}+1\right]\right. \\
& \quad+\left[N_{1}^{4}(1+\mu)^{2}-4 N_{1}^{3}(1+\mu)+2 N_{1}^{2}(3+\mu)-4 N_{1}+1\right\}=0
\end{aligned}
$$

finally leads to

$$
\begin{aligned}
(1- & \left.N_{1} \delta_{1}\right)\left\{-N_{1} \delta_{1}\left[\mu \sqrt{N_{1}^{3} 2}\left(\sqrt{2}-\sqrt{N_{1}}\right)-\left(1-N_{1}\right)^{2}\right]\left[\mu \sqrt{N_{1}^{3} 2}\left(\sqrt{2}+\sqrt{N_{1}}\right)+\left(1-N_{1}\right)^{2}\right]\right. \\
& \left.+\left[\mu N_{1}^{2}+\left(1-N_{1}\right)^{2}\right]^{2}\right\}=0
\end{aligned}
$$

Evidently,

$$
\mu \sqrt{N_{1}^{3}}\left(\sqrt{2}+\sqrt{N_{1}}\right)+\left(1-N_{1}\right)^{2}>0 \quad\left[\mu N_{1}^{2}+\left(1-N_{1}\right)^{2}\right]^{2}>0
$$

and, due to the condition $\kappa>0$, the expression in the first square brackets must be positive to fulfill (3.11). With this, we have the following restrictions on $N_{1}$

$$
N_{1} \delta_{1}<1 \quad \mu \sqrt{N_{1}^{3}}\left(\sqrt{2}-\sqrt{N_{1}}\right)>\left(1-N_{1}\right)^{2}
$$

So, we have

$$
\delta_{1}\left(N_{1}\right)=\frac{2 \mu^{2} N_{1}^{2}}{2 \mu^{2} N_{1}^{3}-\left[\mu N_{1}^{2}+\left(1-N_{1}\right)^{2}\right]^{2}}-\frac{1}{N_{1}}
$$

and now we can find the maximum of $\kappa$, i.e. the minimum of $\varphi\left(N_{1}\right)=N / \delta=N_{1} \delta_{1}\left(N_{1}\right)$

$$
\frac{d \varphi}{d N_{1}}=2 \mu^{2} N_{1}^{2} \frac{(1+\mu)^{2} N_{1}^{4}-2(3+\mu) N_{1}^{2}+8 N_{1}-3}{\left\{2 \mu^{2} N_{1}^{3}-\left[\mu N_{1}^{2}+\left(1-N_{1}\right)^{2}\right]^{2}\right\}^{2}}
$$


The numerator has two real and two complex roots

$$
\frac{\sqrt{4+3 \mu}-1}{1+\mu} \quad-\frac{\sqrt{4+3 \mu}+1}{1+\mu} \quad \frac{1+\mathrm{i} \sqrt{\mu}}{1+\mu} \quad \frac{1-\mathrm{i} \sqrt{\mu}}{1+\mu}
$$

Only the first of them is positive and for any given value of $\mu$ provides the absolute minimum of the function $\varphi\left(N_{1}\right)$.

Substituting $N_{1}=(\sqrt{4+3 \mu}-1) /(1+\mu)$ in (3.13), after simplifications we get

$$
\delta_{1}^{\star}=\frac{8}{3} \frac{16+23 \mu+9 \mu^{2}+2(2+\mu) \sqrt{4+3 \mu}}{64+80 \mu+27 \mu^{2}}
$$

The last expression is obviously positive, so the second inequality in (3.12) holds. Now we verify the first one. It leads to

$$
N=\frac{64}{3} \frac{64+112 \mu+61 \mu^{2}+9 \mu^{3}+\left(64+136 \mu+103 \mu^{2}+27 \mu^{3}\right) \sqrt{4+3 \mu}}{(1+\mu)^{2}\left(64+80 \mu+27 \mu^{2}\right)}<\delta
$$

Eliminating the square root, one may see that

$$
729 \mu^{6}+5778 \mu^{5}+19279 \mu^{4}+32758 \mu^{3}+27809 \mu^{2}+9344 \mu>0
$$

So, for any $\mu>0$ we have $N<\delta$, and (3.12) are fulfilled. Finally, we have

$$
\begin{aligned}
\delta^{\star} & =\frac{8}{3} \frac{16+23 \mu+9 \mu^{2}+2(2+\mu) \sqrt{4+3 \mu}}{(1+\mu)^{2}\left(64+80 \mu+27 \mu^{2}\right)} \\
h^{\star} & =\frac{2}{3} \frac{64+248 \mu+255 \mu^{2}+81 \mu^{3}-2\left(16+20 \mu+9 \mu^{2}\right) \sqrt{4+3 \mu}}{\left(64+80 \mu+27 \mu^{2}\right)(1+\mu)^{3}}
\end{aligned}
$$

and respectively

$$
\begin{aligned}
\eta^{\star} & =2 \sqrt{\frac{2}{3} \frac{16+23 \mu+9 \mu^{2}+2(2+\mu) \sqrt{4+3 \mu}}{(1+\mu)^{2}\left(64+80 \mu+27 \mu^{2}\right)}} \\
\zeta^{\star} & =\sqrt{\frac{64+248 \mu+255 \mu^{2}+81 \mu^{3}-2\left(16+20 \mu+9 \mu^{2}\right) \sqrt{4+3 \mu}}{6\left(64+80 \mu+27 \mu^{2}\right)(1+\mu)^{3}}}
\end{aligned}
$$

which determine the optimal values of stiffness and damping for DVA. For $\mu=0.1$, the curve $f(\gamma)$ in the vicinity of peaks is presented in Figs. 4a and $4 \mathrm{~b}$ for both cases - according to (3.3) and $(3.16)$.
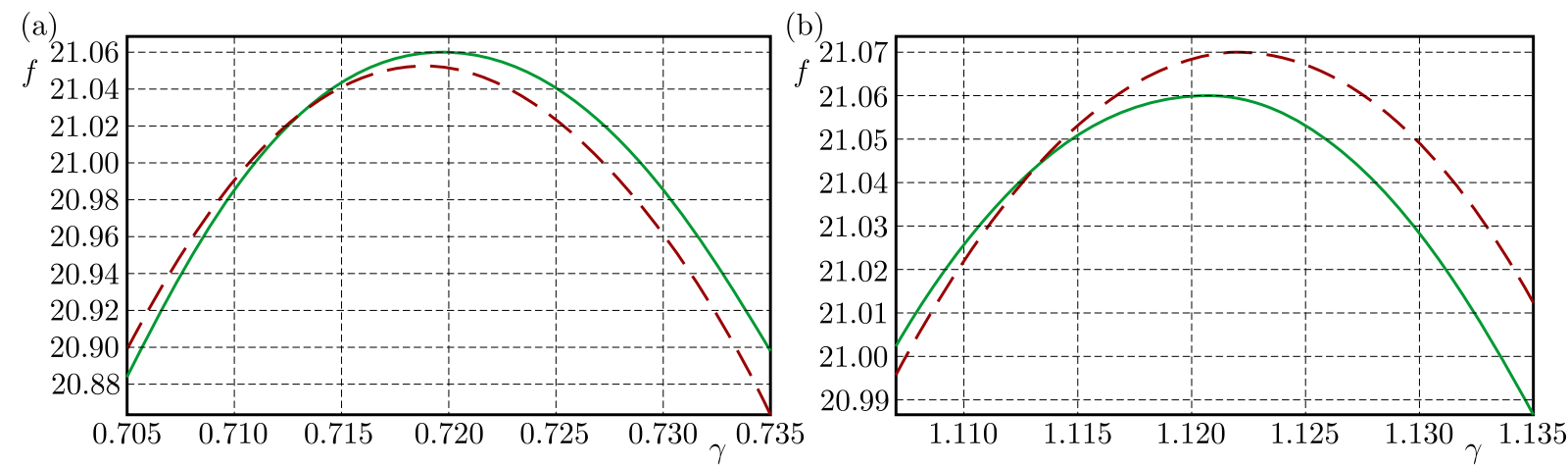

Fig. 4. Frequency-amplitude curve in the neighbourhood of peaks. Long dash line - according to (3.3), solid line - according to (3.16) 
Also, substituting $\delta$ and $N$ into $(3.8)_{2}$, we get

$$
M=-\frac{414 \mu^{2}+1104 \mu+768+\left(54 \mu^{2}+168 \mu+192\right) \sqrt{4+3 \mu}}{9(1+\mu)\left(64+80 \mu+27 \mu^{2}\right)}
$$

The rigorous mathematical proof of the fact that any pair of $\delta, h$ which differs from (3.16) is worse (gives larger maximum of $f$ ) is too cumbersome to be given here. But, at least, this verification versus pair (3.3) is rather simple. Indeed, substituting (3.3) and (3.16) one after the other in (3.4), we determine the extremal values of $\gamma$ for each case separately with the help of Taylor expansions by $\sqrt{\mu}$

$$
\begin{aligned}
& \gamma_{1}^{\text {upon }(3.3)}=1-\frac{\sqrt{2}}{2} \sqrt{\mu}-\frac{7}{8} \mu-\frac{139 \sqrt{2}}{256}(\sqrt{\mu})^{3}+\frac{1797}{2058} \mu^{2}+\ldots \\
& \gamma_{2}^{\text {upon }(3.3)}=1+\frac{\sqrt{2}}{2} \sqrt{\mu}-\frac{7}{8} \mu+\frac{139 \sqrt{2}}{256}(\sqrt{\mu})^{3}+\frac{1797}{2058} \mu^{2}+\ldots \\
& \gamma_{1}^{\text {upon }(3.16)}=1-\frac{\sqrt{2}}{2} \sqrt{\mu}-\frac{7}{8} \mu-\frac{37 \sqrt{2}}{64}(\sqrt{\mu})^{3}+\frac{107}{128} \mu^{2}+\ldots \\
& \gamma_{1}^{\text {upon }(3.16)}=1+\frac{\sqrt{2}}{2} \sqrt{\mu}-\frac{7}{8} \mu+\frac{37 \sqrt{2}}{64}(\sqrt{\mu})^{3}+\frac{107}{128} \mu^{2}+\ldots
\end{aligned}
$$

And then, we may estimate the gain of using the values by (3.16)

$$
\begin{aligned}
\max & f^{\text {upon }(3.3)}-\max f^{\text {upon }(3.16)}=f\left(\gamma_{2}^{\text {upon }(3.3)}\right)-f\left(\gamma_{2}^{\text {upon }(3.16)}\right) \\
= & \frac{5 \sqrt{2}}{256} \sqrt{\mu}+\frac{101}{8192} \mu+\frac{9859 \sqrt{2}}{131072} \mu^{2}+\ldots
\end{aligned}
$$

Numerically, such verification is even easier - we may just combine (3.1) with condition (3.4) for both cases separately and plot the curve

$$
\psi(\mu)=\max f^{u p o n(3.3)}-\max f^{\text {upon }(3.16)}
$$

This curve is presented in Fig. 5, the dash line corresponds to $\gamma_{1}^{\text {upon(3.3) }}$, so the distance between the two branches is the height difference between the two peaks for case (3.3).

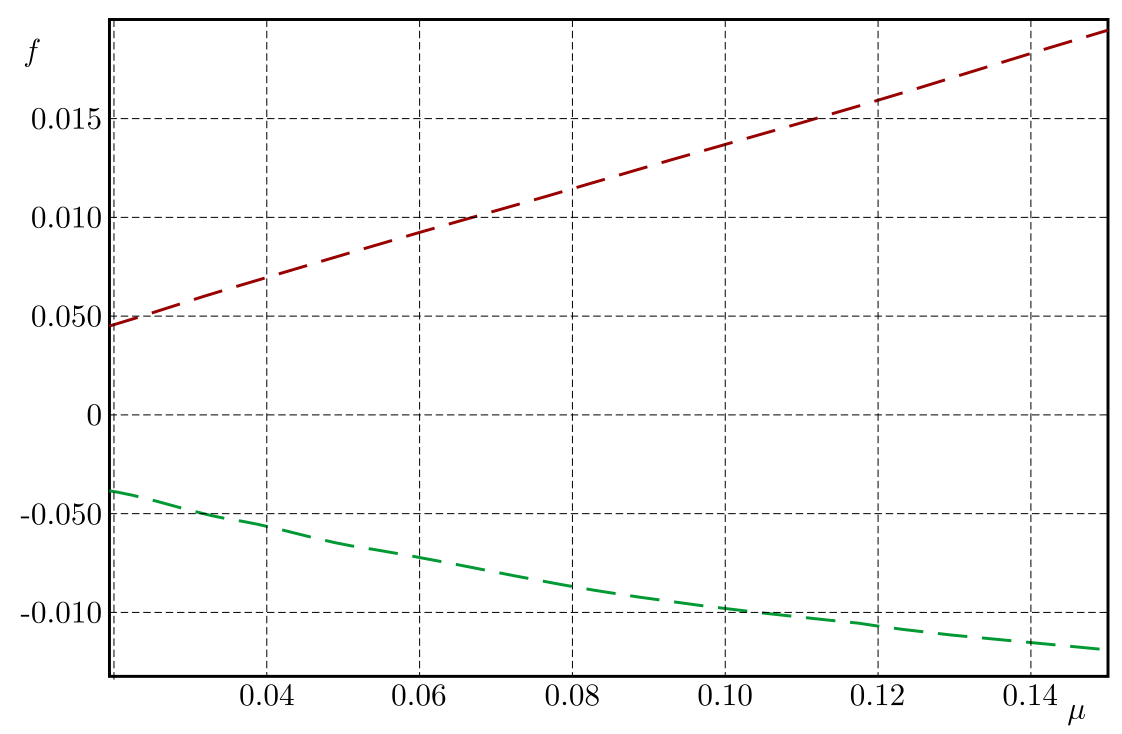

Fig. 5. Difference in $f_{\max }$ values for two cases 
As the last step, let us write down the analytical expression for $\left|X_{p}\right| /\left(X_{p}\right)_{s t}$. Substituting $\delta^{\star}$, $h^{\star}$ according to $(3.16)$ and $\gamma_{2}=\left(-M+\sqrt{M^{2}-4 N}\right) / 2$ in $f$, we have

$$
\left(\frac{\left|X_{p}\right|}{\left(X_{p}\right)_{s t}}\right)^{2}=f^{\star}=\frac{A+B R}{C+D R}
$$

where

$$
\begin{array}{lll}
A=2\left(A_{0}+A_{1} r\right) & B=3(1+\mu) \sigma\left(B_{0}+B_{1} r\right) & C=2\left(C_{0}+C_{1} r\right) \\
D=3(1+\mu) \sigma\left(D_{0}+D_{1} r\right) & R=\sqrt{M^{2}-4 N} & r=\sqrt{4+3 \mu} \\
\sigma=64+80 \mu+27 \mu^{2} & &
\end{array}
$$

Here, the polynomial coefficients are given by formulas

$$
\begin{aligned}
A_{0} & =8192+57856 \mu+145536 \mu^{2}+181000 \mu^{3}+122048 \mu^{4}+43764 \mu^{5}+7083 \mu^{6}+243 \mu^{7} \\
A_{1} & =-4096-8960 \mu-1152 \mu^{2}+13252 \mu^{3}+15443 \mu^{4}+7149 \mu^{5}+1242 \mu^{6} \\
B_{0} & =64+376 \mu+564 \mu^{2}+331 \mu^{3}+69 \mu^{4} \quad B_{1}=-32+4 \mu+63 \mu^{2}+46 \mu^{3}+9 \mu^{4} \\
C_{0} & =\mu^{2}\left(1437696+5792256 \mu+9830592 \mu^{2}+8976744 \mu^{3}+4645404 \mu^{4}+1291545 \mu^{5}\right. \\
& \left.+150903 \mu^{6}\right) \\
C_{1} & =\mu^{2}\left(387072+1278720 \mu+1804896 \mu^{2}+1411020 \mu^{3}+652725 \mu^{4}+170586 \mu^{5}+19683 \mu^{6}\right) \\
D_{0} & =\mu^{2}\left(155713536+906854400 \mu+2321104896 \mu^{2}+3410021376 \mu^{3}+3146726016 \mu^{4}\right. \\
& \left.+1871659224 \mu^{5}+705324672 \mu^{6}+157181148 \mu^{7}+17380089 \mu^{8}+531441 \mu^{9}\right) \\
D_{1} & =\mu^{2}\left(35389440+177389568 \mu+397799424 \mu^{2}+529362432 \mu^{3}+463738176 \mu^{4}\right. \\
& \left.+275945292 \mu^{5}+108628209 \mu^{6}+25572591 \mu^{7}+2716254 \mu^{8}\right)
\end{aligned}
$$

Eliminating the root $R$ from the denominator, we have

$$
\left(A C-B D R^{2}\right)+(B C-A D) R
$$

in the numerator, and multiplier before $R$ must be zero, because of $f\left(\gamma_{2}^{\text {upon }(3.16)}\right)=f\left(\gamma_{1}^{\text {upon }(3.16)}\right)$, i.e. $f^{\star}$ is an even function of $R$ (direct calculation confirms this fact). To simplify the fraction obtained, we also need to eliminate the "small" root $r$ from the denominator. The final expression

$$
\frac{\left|X_{p}\right|}{\left(X_{p}\right)_{s t}}=\sqrt{\frac{(8+9 \mu)^{2}(16+9 \mu)-128 \sqrt{(4+3 \mu)^{3}}}{27 \mu^{2}(32+27 \mu)}}
$$

is a quite remarkable recognition because reduction of the fraction is possible only at the final stage, and the denominator before this reduction is the polynomial of the 48-th(!) order in $\mu$.

\section{Conclusion}

We have discussed the problem of selection of optimal parameters of a DVA according to the classical Den Hartog model (Den Hartog, 1940). We have shown that the solution may be given in an accurate algebraic form which updates somewhat the known result. The analytical approach presented here may be more applicable for solving problems connected with the use of passive damping devices. We have also compared the results of two approaches and determined the error estimation which has been illustrated by the corresponding frequency-amplitude curves. 


\section{References}

1. Brown S., San Diego S. Et Al. (Edit.), 2002, Encyclopedia of Vibrations, Academic Press, $1685 \mathrm{p}$.

2. Brock J.E., 1946, A note on the damped vibration absorber, Journal of Applied Mechanics, 68, A-284

3. Den Hartog J.P., 1940, Mechanical Vibrations, 2nd ed., McGraw-Hill, New York

4. Frahm H., 1911, Device for damping vibration bodies, US Patent No. 989/959, 1911

5. Griffin S., Gussy J., Lane S.A., Henderson B.K., Sciulli D., 2002, Virtual skyhook vibration isolation system, Journal of Vibration and Acoustics, 124, 63-67

6. Hunt J.B., 1979, Dynamic Vibration Absorbers, Mechanical Engineering Publications, London

7. Johnson C.D., 1995, Design of passive damping systems, Journal of Vibration and Acoustics, 117(B), 171-175

8. Korenev B.G., Reznikov L.M., 1993, Dynamic Vibration Absorbers, Wiley, New York

9. LIU K., LIU J., 2005, The damped dynamic vibration absorbers: revisited and new result, Journal of Sound and Vibration, 284, 1181-1189

10. Marano G.C., Greco R., Trentadue F., Chiaia B., Constrained reliability-based optimization of linear tuned mass dampers for seismic control, International Journal of Solids and Structures, 44, 22/23, 7370-7388

11. Mead D.J., 2000, Passive Vibration Control, John Wiley \& Sons Ltd, Chichester, West Sussex, England

12. Ormondroyd J., Den Hartog J.P., 1928, The theory of the vibration absorber, Transactions of the American Society of Mechanical Engineers, 49, A9-22

13. Pozdniakovich A.E., Puzyrov V.E., 2009, On the choice of the parameters of the dynamical vibration absorber, Mekhanika Tverdogo Tela, 39, 167-172

14. Sun J.Q., Jolly M.R., Norris M.A., 1995, Passive, adaptive and active tuned vibration absorbers survey, Transactions of the ASME, 117, 4, 234-242

15. Viet L.D., Anh N.D., Matsuhisa H., 2011, The effective damping approach to design a dynamic vibration absorber using Coriolis force, Journal of Sound and Vibration, 330, 1904-1916

16. Warburton G.B., Ayorinde E.O., 1980, Optimum absorber parameters for simple systems, Earthquake Engineering and Structural Dynamics, 8, 197-217 